\title{
Hsp90 Detection by Two Strategies based on AuNCs' Fluorescence
}

\author{
Maria V. Romeo, Antonio Aires, Jesús Berganza, and Aitziber L. Cortajarena \\ GAIKER Technology Centre \\ Parque Tecnológico, Ed. 202, 48170 Zamudio, Bizkaia, Spain \\ villuendas@gaiker.es; berganza@gaiker.es \\ CIC biomaGUNE \\ Paseo Miramón 182, 20014, Donostia-San Sebastián, Spain \\ mvilluendas.gaiker@cicbiomagune.es; aaires@cicbiomagune.es; alcortajarena@cicbiomagune.es
}

\section{Extended Abstract}

Fluorescent gold nanoclusters (AuNCs) are emerging in biomedical and biosensing applications due to its photophysical properties linked to their small size. The aim of this study is to fabricate a biosensor based on the fluorescence of gold nanoclusters stabilized in repeat proteins, in particular tetratricopeptide repeat (TPR) proteins (AuNCs@ proteins) which are genetically modified by the addition of two different recognition modules (CTPR390 and TPR2A). This modification allows the specific interaction between nanoclusters and analyte, Hsp90, which is a biomarker of myocardial fibrosis, a heart disease [1].

Hsp90 is present in blood serum at very low concentration (0.31 nM). Supposing a ratio AuNCs@ protein:Hsp90 1:1 in assays, the first objective is to optimize AuNCs@protein synthesis [2], [3] conditions until $0.2 \mathrm{nM}$ can be detected by fluorescence. The detection limit is reached by a modification of incubation conditions [4] and molar ratio of components that take part on the synthesis reaction.

The final goal of this project is to fabricate a biosensor and there are two possibilities considered: lateral flow [5] measuring test and control line's fluorescence by a portable fluorescence lector, and/or measuring fluorescence directly in solution.

Measuring directly in solution, first a calibration curve was obtained to determine the limit of detection $(\mathrm{LoD}=11.03$ $\mathrm{nM})$ and limit of quantification (LoQ $=36.77 \mathrm{nM})$ of the system by measuring the fluorescence intensity at different concentrations $(\lambda \mathrm{exc}=320 \mathrm{~nm}$ and $\lambda \mathrm{em}=400 \mathrm{~nm})$. According to these limits, Hsp90 aliquots were added to a AuNCs $@$ protein solution. Fluorescence was measured before and after the addition in order to determine if there was a variation promoted by the interaction with Hsp90. However, the non-specific effect of the plasma proteins on the fluorescence signal did not allow the quantification of the analyte by this method.

Therefore, other detection formats were studied, such as microplate system. For that purpose, ELISA experiments were developed to mimic the immobilization strategy of lateral flow's system. In this case, AntiHsp90 immobilized on the plate would capture the analyte (Hsp90) and the AuNCs@protein, that also recognizes Hsp90, is proposed as a developing agent, being its fluorescence the readout signal related to the amount of Hsp90 captured. BSA was used in the control experiments instead of the analyte, to evaluate the possibility of unspecified interactions. First of all, direct immobilization of TPR proteins and Hsp90 was developed to fix detection limits. These experiments revealed that the maximum concentration that can be immobilized due to well's diameter is $20 \mathrm{nM}$ for TPR proteins (AuNCs' templates) and $5 \mathrm{nM}$ for Hsp90. Considering sensitisation step's efficiency (36.20\%), the real concentrations per well were $7.24 \mathrm{nM}$ and $1.81 \mathrm{nM}$, respectively. Therefore, the detection limit of the direct fluorescence of the AuNCs@ protein in microplate assay is not sufficient for the required Hsp90 detection.

In order to improve both systems, some strategies are proposed, such as the use of recognition modules with higher affinity for the analyte. In addition, isolation systems based on magnetic beads can avoid interferences in complex samples; and finally, signal amplification systems can be implemented as developing agents to improve LoD and LoQ. 


\section{References}

[1] Raquel García, David Merino, Jenny M. Gómez, J. Francisco Nistal, María A. Hurlé, Atiziber L. Cortajarena, Ana V. Villar, "Extracellular heat shock protein 90 binding to TGF $\beta$ receptor I participates in TGF $\beta$-mediated collagen production in myocardial fibroblasts," Cellular Signalling.

[2] Pierre Couleaud, Sergio Adan-Bermudez, Antonio Aires, Sara H. Mejies, Begoña Sot, Alvaro Somoza, and Aitziber L. Cortajarena, "Designed Molecular Proteins as Scaffolds of Stabilize Fluorescent Nanoclusters," Biomacromolecules, vol. 16, pp. 3836-3844.

[3] A. Aires, I. Llarena, M. Moller, J. Castro-Smirnov, J. Cabanillas-Gonzalez, A. L. Cortajarena, "A simple approach to design proteins for the sustainable synthesis of metal nanoclusters," Angew. Chem. Int., 2019.

[4] Tiankai Chen, Qiaofeng Yao, Xun Yuan, Ricca Rahman Nasaruddin, and Jianping Xie, "Heating or Cooling: Temperature Effects on the Synthesis of Atomically Precise Gold Nanoclusters," The Journal of Physical Chemistry, vol. 121, pp. 10743-10751.

[5] Xue Li, Xiaoqun Gong, Bo Zhang, Yajuan Liu, Jin Chang and Xuening Zhang, "Ultrasensitive lateral-flow assays based on quantum dot encapsulation with signal amplification," J. Nanoparticles Research, vol. 20, p. 139.

[6] Aitziber L. Cortajarena, Tommi Kajander, Weilan Pan, Melanie J. Cocco and Lunne Regan, "Protein design to understand peptide ligand recognition by tetratricopeptide repeat protein," PEDS, vol. 17, pp. 399-409, 2004.

[7] A. L. Cortajarena, F. Yi F, L. Regan, "Designed TPR modules as novel anticancer agents," ACS Chem Biol., vol. 3, no. 3, pp. 161-6, 2008. 